\title{
Developmental Regression, Depression, and Psychosocial Stress in an Adolescent with Down Syndrome
}

\author{
David S. Stein, PsyD ${ }^{\star}$, Kerim M. Munir, MD, MPH, DSc ${ }^{\star}$, Andrea J. Karweck, PsyD, Emily J. \\ Davidson, MD, $\mathbf{M P H}^{*}$, and Martin T. Stein, $\mathbf{M D}^{\dagger}$ \\ “Developmental Medicine Center, Boston Children's Hospital, Harvard Medical School, Boston, \\ MA \\ †Department of Pediatrics, University of California, San Diego, San Diego, CA
}

\section{Abstract}

CASE: Kristen is a 13-year-old girl with Down syndrome (DS) who was seen urgently with concerns of cognitive and developmental regression including loss of language, social, and toileting skills. The evaluation in the DS clinic focused on potential medical diagnoses including atlantoaxial joint instability, vitamin deficiency, obstructive sleep apnea (OSA), and seizures. A comprehensive medical evaluation yielded only a finding of moderate OSA. A reactive depression was considered in association with several psychosocial factors including moving homes, entering puberty/onset of menses, and classroom change from an integrated setting to a self-contained classroom comprising unfamiliar peers with behavior challenges.

Urgent referrals for psychological and psychiatric evaluations were initiated. Neuropsychological testing did not suggest true regression in cognitive, language, and academic skills, although decreases in motivation and performance were noted with a reaction to stress and multiple environmental changes as a potential causative factor. Psychiatry consultation supported this finding in that psychosocial stress temporally correlated with Kristen's regression in skills.

Working collaboratively, the team determined that Kristen's presentation was consistent with a reactive form of depression (DSM-IV-TR: depressive disorder, not otherwise specified). Kristen's presentation was exacerbated by salient environmental stress and sleep apnea, rather than a cognitive regression associated with a medical cause. Treatment consisted of an antidepressant medication, continuous positive airway pressure for OSA, and increased psychosocial supports. Her school initiated a change in classroom placement. With this multimodal approach to evaluation and intervention, Kristen steadily improved and she returned to her baseline function.

\section{Index terms}

Down syndrome; developmental regression; depression; obstructive sleep apnea

\section{DISCUSSION}

\section{David S. Stein, PsyD, Kerim M. Munir, MD, MPH, DSc, Andrea J. Karweck, PsyD}

Individuals with Down syndrome (DS) and/or intellectual disability (ID) often do not express emotional distress through the usual channels. Rather than exhibiting sadness or irritability as often seen in typically developing peers, children and youth with ID and

Copyright $@ 2013$ Lippincott Williams \& Wilkins

Disclosure: The authors declare no conflict of interest. 
emotional challenges often exhibit a more complex presentation with loss of functional skills including speech, toilet control, and sleep. ${ }^{1}$ In the context of multiple, potential medical conditions associated with DS, it is understandable that the first concern of parents and clinicians is biomedical, including the possibility of early onset cognitive decline or juvenile onset dementia despite a low prevalence in early adolescence. As illustrated in this case, we initiated a multimodal approach to begin the evaluation of developmental regression in Kristen.

An important principle for behaviorally focused clinicians is to consider a behavioral presentation as a form of communication. This is particularly salient for individuals with developmental disabilities who are unlikely to express distress verbally or to be able to use abstract thinking skills to connect experiences with thoughts and emotions. In this case, we learned that Kristen's significant change in behavior was a form of communication. Kristen informed us through the changes in her functioning that the shift in her classroom placement and subsequent loss of relationships with more social and verbal peer models was a considerable challenge for her. Furthermore, the stress of a pending family move, effects of sleep apnea, and onset of puberty and menses added additional psychological burdens to her well-being. Because of Kristen's DS and ID and compounded by her significant withdrawal and limited language, it was not possible to ask her directly about the impact of these experiences. Rather, we were required to use assessment tools, including comprehensive medical, neuropsychological, and psychiatric methods, to rule out other causes and take our best guess that this change in functioning was an expression of depression. To test this assumption, we implemented a treatment plan based on our hypothesis and waited to observe the outcome of multifaceted interventions.

For clinicians working with individuals with disabilities and complex medical conditions such as DS, the impact of psychosocial stress may be overlooked when we are considering potentially more insidious biological causes. However, we must remember that individuals with ID, and particularly those with DS who are often hypersocial based on their neurobiology, are consequently also exquisitely sensitive to changes in their social environment. ${ }^{3,4}$ As children with DS enter adolescence and young adulthood, externalizing problems tend to diminish, but internalizing problems such as depression increase, particularly in the face of psychosocial stress. $5,6 \mathrm{We}$, therefore, encourage clinicians to consider depression and adjustment difficulties in response to social and environmental changes as a potential factor when assessing losses in functioning in individuals with DS and/or ID. Not doing so may lead to incorrect diagnostic assumptions, unnecessary delays in treatment, or interventions that are unlikely to address the causative factor of regression.

\section{References}

1. Cooper SA, Callacott RA. Clinical features and diagnostic criteria of depression in Down's syndrome. Br J Psychiatry. 1994; 165:399-403. [PubMed: 7994516]

2. Holland AJ, Hon J, Huppert FA, Stevens F. Incidence and course of dementia in people with Down's syndrome: findings from a population-based study. J Intellect Disabil Res. 2000; 44:138146. [PubMed: 10898377]

3. Forness SR, Polloway EA. Physical and psychiatric diagnoses of pupils with mild mental retardation currently being referred for related services. Educ Train Ment Retard. 1987; 22:221-228.

4. Brickell C, Munir K. Grief and its complications in individuals with intellectual disability. Harv Rev Psychiatry. 2008; 16:1-12. [PubMed: 18306095]

5. Dykens EM, Shah B, Sagun J, Beck T, King BH. Maladaptive behaviour in children and adolescents with Down's syndrome. J Intellect Disabil Res. 2002; 46:484-492. [PubMed: 12354319] 
6. Dykens EM, Kasari C. Maladaptive behavior in children with Prader-Willi syndrome, Down syndrome, and non-specific mental retardation. Am J Ment Retard. 1997; 102:228-237. [PubMed: 9394132]

\section{Emily J. Davidson, MD, MPH}

Down syndrome (DS) is a risk factor for the development of depression. ${ }^{1}$ Kristen's medical history revealed additional biological events that may adversely affect brain development; she was delivered preterm at 31.5 weeks and she experienced hypoxia associated with a near drowning at age 6 years. The interplay of multiple biological and psychosocial factors in the development of depression for individuals with DS should lead to a thorough medical evaluation and psychological evaluation in this case.

Behavioral changes may be the first or only symptom of a medical problem appreciated by families and clinicians. Thyroid dysfunction may occur in children with DS who are either asymptomatic or present with behavioral concerns such as depression. Pain or other abnormal sensations may be seen in patients with DS who are also at risk for celiac disease, constipation, atlantoaxial instability, and leukemia. Children with intellectual disability (ID) may not be able to identify or report their discomfort. Children with DS also have an increased risk for a seizure disorder and vision and hearing problems, which may affect classroom performance and social interactions and lead to challenging behaviors. Lower metabolic rate and lower activity levels can contribute to obesity, which may also contribute to social isolation and increased risk of depression.

Obstructive sleep apnea (OSA) is common in children with DS, with a prevalence of $30 \%$ to $60 \%$ and may not be obvious to parents. ${ }^{2}$ Symptoms often persist even after tonsillectomy and adenoidectomy. ${ }^{3}$ In addition to the impact of OSA on physical health (e.g., pulmonary hypertension, metabolic syndrome, and obesity), OSA can have a significant impact on school function and mental health. Increased rates of depressive symptoms and impairments in quality of life have been documented in children with $\mathrm{OSA}^{4}$ and sleep apnea has also been linked to poor school performance. ${ }^{5}$

In the general population, higher rates of depression are observed in individuals with OSA and sleep apnea is more common in individuals with depression. Current studies demonstrate neural injury associated with both OSA and depression. ${ }^{6}$ Irritability, depressive symptoms, and poor school function could also serve to increase social isolation leading to further social withdrawal and more difficulty in the school setting. In addition to treating Kristen's depression, treatment for sleep apnea was initiated with continuous positive airway pressure (CPAP). After an initial period of acclimation to the CPAP mask, Kristen preferred sleeping with CPAP, and her parents noted an increase in her energy level.

In this case, an interdisciplinary evaluation led to a clearer understanding of Kristen's presentation. The treatment of her depression and sleep apnea concurrently was associated with steady gains in function. Clinicians who care for children with DS and/or ID are encouraged to consider the interplay of biological and psychosocial factors when they evaluate and manage significant changes in the behavior.

\section{References}

1. McGuire DE, Chicoine B. Depressive disorders in adults with Down syndrome. Habilitat Ment Healthcare Newslett. 1996; 151:26-27.

2. Shott SR, Amin R, Chini B, Heubi C, Hotze S, Akers R. Obstructive sleep apnea: should all children with Down syndrome be tested? Arch Otolaryngol Head Neck Surg. 2006; 132:432-436. [PubMed: 16618913] 
3. Donnelly LF, Shott SR, LaRose CR, Chini BA, Amin RS. Causes of persistent obstructive sleep apnea despite previous tonsillectomy and adenoidectomy in children with Down syndrome as depicted on static and dynamic cine MRI. AJR Am J Roentgenol. 2004; 183:175-181. [PubMed: 15208134]

4. Crabtree VM, Varni JW, Gozal D. Health-related quality of life and depressive symptoms in children with suspected sleep-disordered breathing. Sleep. 2004; 27:1131-1138. [PubMed: 15532207]

5. Gozal D. Sleep-disordered breathing and school performance in children. Pediatrics. 1998; 102:616620. [PubMed: 9738185]

6. Cross RL, Kumar R, Macey PM, et al. Neural alterations and depressive symptoms in obstructive sleep apnea patients. Sleep. 2008; 31:1103-1109. [PubMed: 18714782]

\section{Martin T. Stein, MD}

A unifying theme of the Challenging Case series in the Journal has been the value of a biopsychosocial perspective to organize our thinking about diagnosis and management of neurodevelopmental and behavioral conditions. ${ }^{1}$ The case of Kristen, a 13-year-old child with Down syndrome (DS) who experienced a regression of cognitive and social skills, is an instructive example of how the biopsyschosocial model can be useful.

Both commentaries emphasized the importance of simultaneous consideration of biological and psychosocial factors. I have found it useful to simplify the model by beginning all medical evaluations with a perspective that biological, psychological, and social considerations should be assessed initially with equal status. In this way, data that support or refute a condition that belongs to each of these areas will not be lost in the diagnostic process. As data are accumulated from the patient's history, physical examination, and laboratory tests, 1, 2, or all 3 areas may take on a greater significance. For Kristen, biological (obstructive sleep apnea [OSA]), psychological (depression), and social (a new school environment) influences came together to bring about the behavioral changes. A different management strategy for each condition was necessary for a successful outcome.

Clinical recognition of a behavioral diagnosis in a child or adolescent with a neurodevelopmental condition may be difficult even for an experienced clinician. A single case that occurred 25 years ago provided me with a new insight into this problem. Lucia was a 10-year-old child with DS. Her teacher and the school nurse were convinced that Lucia's daily hyperactivity in the classroom was caused by attention-deficit hyperactivity disorder (ADHD). Before evaluating Lucia, I had never considered ADHD in a child with an intellectual deficiency. Either my teachers or my clinical experience suggested that the behaviors of ADHD could not be differentiated from behaviors seen in many intellectually challenged children. Using standard ADHD diagnostic criteria, I discovered that her behaviors in class and at home were consistent with ADHD. A trial of a stimulant medication brought about dramatic improvement in Lucia's hyperactivity; classroom learning and social behaviors at school and home improved. Since that time, small randomized-controlled trials support the value of a stimulant medication for ADHD in other neurodevelopmental conditions including fetal alcohol syndrome, Williams syndrome, autistic spectrum disorder, fragile $\mathrm{X}$ syndrome, Turner syndrome, and Tourette syndrome. ${ }^{2}$

An even greater challenge was the assessment of depression in a patient with intellectual deficiency and limited communication capacity. How can the feeling of sadness and the experience of anhedonia be established in patients similar to Kristen? In this case, the multidisciplinary team developed a hypothesis that a reactive depression secondary to psychosocial stress contributed to her behaviors. To test the hypothesis, an antidepressant was prescribed along with treatment for OSA, a change in school environment and psychosocial support. One final comment: I would have asked Kristen to draw a picture of 
her family, of her school, or herself. Even with her limited cognitive ability, a drawing may have revealed a clue to her emotional state. ${ }^{3}$

\section{References}

1. Engel GL. The need for a new medical model: a challenge for biomedicine. Science. 1977; 106:129-136. [PubMed: 847460]

2. Hagerman, RJ. Neurodevelopmental Disorders: Diagnosis and Treatment. New York, NY: Oxford University Press; 1999.

3. Stein MT. The use of family drawings by children in pediatric practice. J Dev Behav Pediatr. 1997; 18:334-339. [PubMed: 9349977] 